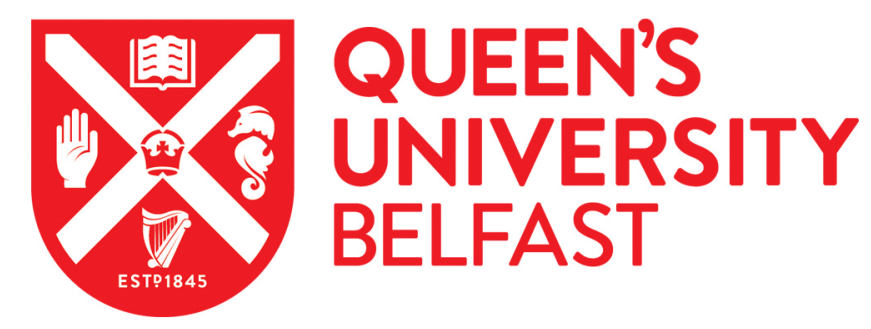

\title{
Sudden death and sudden birth of entanglement in common structured reservoirs
}

Mazzola, L., Maniscalco, S., Piilo, J., Suominen, K. -A., \& Garraway, B. M. (2009). Sudden death and sudden birth of entanglement in common structured reservoirs. Physical Review A (Atomic, Molecular, and Optical Physics), 79(4), [042302]. https://doi.org/10.1103/PhysRevA.79.042302

Published in:

Physical Review A (Atomic, Molecular, and Optical Physics)

Document Version:

Publisher's PDF, also known as Version of record

Queen's University Belfast - Research Portal:

Link to publication record in Queen's University Belfast Research Portal

Publisher rights

(C) 2009 The American Physical Society

\section{General rights}

Copyright for the publications made accessible via the Queen's University Belfast Research Portal is retained by the author(s) and / or other copyright owners and it is a condition of accessing these publications that users recognise and abide by the legal requirements associated with these rights.

Take down policy

The Research Portal is Queen's institutional repository that provides access to Queen's research output. Every effort has been made to ensure that content in the Research Portal does not infringe any person's rights, or applicable UK laws. If you discover content in the Research Portal that you believe breaches copyright or violates any law, please contact openaccess@qub.ac.uk. 


\title{
Sudden death and sudden birth of entanglement in common structured reservoirs
}

\author{
L. Mazzola, ${ }^{1}$ S. Maniscalco, ${ }^{1, *}$ J. Piilo, ${ }^{1}$ K.-A. Suominen, ${ }^{1}$ and B. M. Garraway ${ }^{2}$ \\ ${ }^{1}$ Department of Physics and Astronomy, University of Turku, FI-20014 Turun yliopisto, Finland \\ ${ }^{2}$ Department of Physics and Astronomy, University of Sussex, Falmer, Brighton BN1 9QH, United Kingdom
}

(Received 19 December 2008; published 1 April 2009)

\begin{abstract}
We study the exact entanglement dynamics of two qubits in a common structured reservoir. We demonstrate that for certain classes of entangled states, entanglement sudden death occurs, while for certain initially factorized states, entanglement sudden birth takes place. The backaction of the non-Markovian reservoir is responsible for revivals of entanglement after sudden death has occurred, and also for periods of disentanglement following entanglement sudden birth.
\end{abstract}

DOI: $10.1103 /$ PhysRevA.79.042302

PACS number(s): 03.67.Bg, 03.65.Ud, 03.65.Yz, 42.50.-p

Entanglement is one of the most intriguing features of quantum mechanics [1]: hence understanding its properties and dynamics is of paramount relevance for a number of applications in modern physics. Furthermore, quantum cryptography, quantum information processing, and quantum measurement are examples of branches of physics in which entanglement plays an essential role [2]. Quantum properties, however, are very fragile: realistic quantum systems are not closed, and due to the interaction with the environment, their entanglement and coherence can be irretrievably lost. On the other hand, recent works [3-5] have shown that entanglement can actually revive, or be preserved, using the quantum Zeno effect, or it can even be trapped. Such effects arise when the system of interest interacts with non-Markovian reservoirs. These reservoirs are characterized by structured spectral distributions [6].

In this paper we propose an exactly solvable model for the time evolution of two qubits interacting with a common structured reservoir. We investigate the entanglement dynamics for initially entangled states of the qubits, and also for initially factorized states. We identify the effects of the nonMarkovian backaction of the reservoir during the disentangling process and in the entanglement birth phenomenon. In particular we prove that, due to the reservoir memory effect, entanglement sudden death (ESD) is enhanced as compared to the Markovian case. Contrary to a previous study on ESD in common environments [7], our approach is exact and it does not rely on either the Born or the Markov approximation.

The entanglement dynamics of two qubits interacting with two independent Lorentzian reservoirs is known [3]. In this paper we focus on the common reservoir scenario and compare it with the independent reservoirs case. We note that, already in the Markovian case, the entanglement dynamics in common reservoirs and that in independent reservoirs present striking differences. While two qubits interacting with two independent reservoirs can disentangle completely and permanently in a finite time [8,9], in a common reservoir entanglement can disappear for a finite time and then reappear again [10]. This is due to the fact that common reservoirs tend to create entanglement rather than destroy it com-

\footnotetext{
*sabrina.maniscalco@utu.fi
}

pletely, since they indirectly couple the two qubits [11]. As we show in the following, even when the qubits are initially prepared in a factorized state, the correlation created by the environment can lead to a phenomenon which is known as the entanglement sudden birth (ESB) $[12,13]$.

The analytic solution presented in this paper is valid for a general initial state of the two-qubit system, and includes states with two excitations. The exact solution for the singleexcitation case was discussed in Ref. [4], where it was shown that the initial entanglement never disappears for finite periods of time; i.e., ESD never occurs. When two excitations are present, as for the model discussed here, the derivation of the exact analytical solution is much more complicated. We have proved, however, that by using the pseudomode approach [14] and establishing a connection with a three-level ladder system [15], it is still possible to solve the dynamics without performing any approximations.

We consider a two-qubit system interacting with a common zero-temperature bosonic reservoir. Our chosen specific system consists of two two-level atoms interacting with the electromagnetic field. The initial state of the multimode field is the vacuum state. The Hamiltonian of such a system in the rotating wave approximation is given by $H=H_{0}+H_{\text {int }}$, which, in the basis $\{|00\rangle,|10\rangle,|01\rangle,|11\rangle\}$, reads

$$
\begin{gathered}
H_{0}=\omega_{0}\left(\sigma_{+}^{A} \sigma_{-}^{A}+\sigma_{+}^{B} \sigma_{-}^{B}\right)+\sum_{k} \omega_{k} a_{k}^{\dagger} a_{k}, \\
H_{\mathrm{int}}=\left(\sigma_{+}^{A}+\sigma_{+}^{B}\right) \sum_{k} g_{k} a_{k}+\text { H.c. }
\end{gathered}
$$

Here, $\sigma_{ \pm}^{A}$ and $\sigma_{ \pm}^{B}$ are, respectively, the Pauli raising and lowering operators for atoms $A$ and $B, \omega_{0}$ is the Bohr frequency of the two atoms, $a_{k}$ and $a_{k}^{\dagger}$ are the annihilation and creation operators for the field mode $k$, and mode $k$ is characterized by the frequency $\omega_{k}$ and the coupling constant $g_{k}$. For the sake of simplicity, in the following we assume that the two atoms interact resonantly with a Lorentzian structured reservoir, such as, e.g., the electromagnetic field inside a lossy resonator.

Since the atoms are identical and equally coupled to the reservoir, the dynamics of the two qubits can be effectively described by a four-state system in which three states are coupled to the vacuum in a ladder configuration, and one 
state is completely decoupled from the other states and from the field. This can be shown by writing the Hamiltonian in the basis $\{|0\rangle=|00\rangle,|+\rangle=(|10\rangle+|01\rangle) / \sqrt{2},|-\rangle$ $=(|10\rangle-|01\rangle) / \sqrt{2},|2\rangle=|11\rangle\}$ so that

$$
\begin{gathered}
H_{0}=2 \omega_{0}|2\rangle\langle 2|+\omega_{0}(|+\rangle\langle+|+|-\rangle\langle-|)+\sum_{k} \omega_{k} a_{k}^{\dagger} a_{k}, \\
H_{\text {int }}=\sum_{k} \sqrt{2} g_{k} a_{k}(|+\rangle\langle 0|+| 2\rangle\langle+|)+\text { H.c. }
\end{gathered}
$$

The $|+\rangle$ and $|-\rangle$ states are, respectively, the super-radiant and subradiant states. From Eqs. (3) and (4) it is apparent that the subradiant state does not decay. On the contrary, the superradiant state is coupled to states $|0\rangle$ and $|2\rangle$ via the electromagnetic field.

The total Hamiltonian, given by Eqs. (3) and (4), actually consists of two parts, a part describing the free dynamics of the $|-\rangle$ state, and the remaining terms describing a three-state ladder system $\{|0\rangle,|+\rangle,|2\rangle\}$ with the transitions $|0\rangle \leftrightarrow|+\rangle$ and $|+\rangle \leftrightarrow|2\rangle$ having the same frequencies and identically coupled with the common bosonic reservoir.

It is a mathematically and computationally demanding task to solve numerically the infinite set of differential equations for the complex amplitudes appearing in the state vector of the total system. However, having in mind Eqs. (3) and (4), we can greatly simplify the analytical treatment by noting that the dynamics of the three-level ladder system, characterized by transitions with equal frequencies and identically coupled to the same Lorentzian structured reservoir, can be exactly solved using the pseudomode approach [14]. In Ref. [15] this approach is extended to multiple excitations.

The pseudomodes are auxiliary variables defined from the properties of the spectral distribution, and they allow us to derive a Markovian master equation for the extended system comprised of the system of interest and the pseudomodes. Such an exact master equation describes the coherent interaction between the system and the pseudomodes, and the latter, in turn, leak into independent Markovian reservoirs.

The exact dynamics of the two atoms interacting with a Lorentzian structured reservoir is contained in the following pseudomode master equation:

$$
\frac{\partial \tilde{\rho}}{\partial t}=-i[V, \tilde{\rho}]-\frac{\Gamma}{2}\left(a^{\dagger} a \tilde{\rho}+\tilde{\rho} a^{\dagger} a-2 a \tilde{\rho} a^{\dagger}\right),
$$

where $\tilde{\rho}$ is the density matrix of the pseudomode plus the atom, and

$$
V=\sqrt{2} \Omega\left(a|+\rangle\left\langle 0\left|+a^{\dagger}\right| 0\right\rangle\langle+|+a| 2\rangle\left\langle+\left|+a^{\dagger}\right|+\right\rangle\langle 2|\right) .
$$

Here, $a$ and $a^{\dagger}, \Gamma$, and $\Omega$ are, respectively, the annihilation and creation operators, the pseudomode decay rate into its Markovian reservoir, and the coupling constant of the pseudomode to the ladder system. The pseudomode is associated with the Lorentzian spectral distribution

$$
J(\omega)=\frac{\Omega^{2}}{2 \pi} \frac{\Gamma}{\left(\omega-\omega_{0}\right)^{2}+(\Gamma / 2)^{2}},
$$

where $\Gamma / 2$ describes the frequency width of the spectrum and is related to the reservoir correlation time. For small values of $\Gamma$ the pseudomode can be associated with the real cavity mode of frequency $\omega_{0}$.

In order to find the dynamics of the two atoms, we solve the master equation in Eqs. (5) and (6). We focus on the case in which the total system contains at most two excitations. In this case we need to solve a set of 64 differential equations obtained from Eqs. (5) and (6). The symmetry properties of the system allow grouping of the set of differential equations into decoupled subset of differential equations of smaller size. For each subset we solve the associated system of algebraic equations in the Laplace transform space. Tracing out the pseudomode degree of freedom, we obtain the density matrix of the reduced atomic system for every initial atomic state.

Our aim is to investigate the effects of the nonMarkovianity of the reservoir on atomic entanglement dynamics. To quantify the entanglement we use the Wootters concurrence [16], defined as $C(t)$ $=\max \left\{0, \sqrt{\lambda_{1}}-\sqrt{\lambda_{2}}-\sqrt{\lambda_{3}}-\sqrt{\lambda_{4}}\right\}$, where $\left\{\lambda_{i}\right\}$ are the eigenvalues of the matrix $R=\rho\left(\sigma_{y}^{A} \otimes \sigma_{y}^{B}\right) \rho^{*}\left(\sigma_{y}^{A} \otimes \sigma_{y}^{B}\right)$, with $\rho^{*}$ denoting the complex conjugate of $\rho$ and $\sigma_{y}^{A / B}$ are the Pauli matrices for atoms $A$ and $B$. This quantity attains its maximum value of 1 for maximally entangled states and vanishes for separable states.

For initial states of the form

$$
|\Psi(0)\rangle=\alpha|00\rangle+e^{i \theta}\left(1-\alpha^{2}\right)^{1 / 2}|11\rangle
$$

the density matrix of the atomic system has an " $\mathrm{X}$ " form, with nonzero elements only along the main diagonal and antidiagonal. Due to the structure of the differential equations for the density-matrix elements [see Eq. (5)], the $\mathrm{X}$ form is preserved during the evolution. Then the concurrence has a simple analytic expression

$$
C(t)=2 \max \{0,|w(t)|-\sqrt{b(t) c(t)},|z(t)|-\sqrt{a(t) d(t)}\},
$$

where $a(t)=\rho_{00 ; 00}, \quad b(t)=\rho_{10 ; 10}, \quad c(t)=\rho_{01 ; 01}, \quad d(t)=\rho_{11 ; 11}$, $w(t)=\rho_{00 ; 11}, z(t)=\rho_{10 ; 01}$, and $\rho_{i j ; k l}=\langle i, j|\rho| k, l\rangle$ are the elements of the atomic density matrix $\rho$.

We plot the time evolution of concurrence as a function of both the parameter $\alpha^{2}$ and the dimensionless quantity $\gamma_{0} t$, where the parameter $\gamma_{0}=4 \Omega^{2} / \Gamma$ is the Markovian decay rate of the atoms, i.e., the inverse of the atomic relaxation time in the Markovian limit [6]. The parameters $\Gamma$ and $\Omega$ are also expressed in units of $\gamma_{0}$. We look at the dynamics in the strong-coupling regime, which is obtained when $\Gamma / \Omega<4$; in particular we choose $\Gamma=0.2 \gamma_{0}$ and $\Omega=\sqrt{0.05} \gamma_{0}$, corresponding to $\Gamma / \Omega=\sqrt{0.8}$. These values are achievable experimentally, e.g., with circuit QED setups [17].

Figure 1(a) shows the behavior of concurrence for two qubits prepared in state (8). Depending on $\alpha^{2}$ different dynamical behavior is clearly visible. For $\alpha^{2} \gtrsim 1 / 4$ the entanglement dynamics presents damped oscillations. For $\alpha^{2} \lesssim 1 / 4$ finite periods of complete disentanglement are followed by entanglement revivals. Entanglement revivals are amplified for stronger non-Markovian conditions, i.e., for smaller values of the ratio $\Gamma / \Omega$. We have verified that when the ratio $\Gamma / \Omega$ is, e.g., ten times smaller than the one used in Fig. 1, the oscillations become much stronger in amplitude, the number of ESD periods and revivals increases, and they 


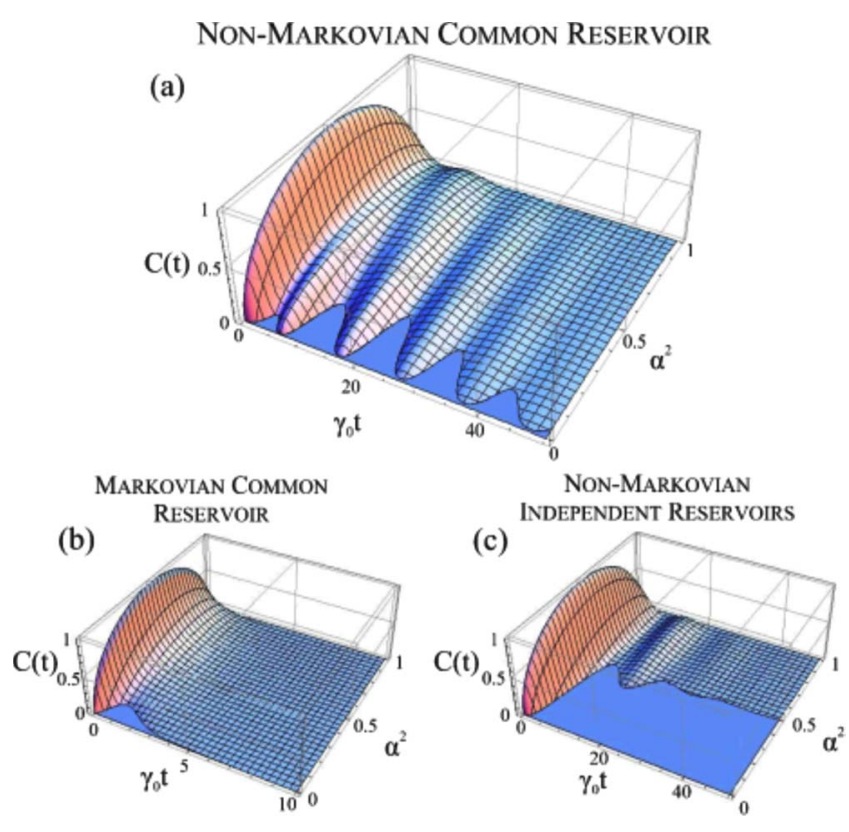

FIG. 1. (Color online) (a) Concurrence as a function of scaled time and $\alpha^{2}$ for two atoms prepared in state (8) and interacting with a common Lorentzian structured reservoir. For comparison we show also (b) the equivalent Markovian case and (c) the nonMarkovian independent reservoirs situation.

last for a longer time. On the other hand, when we increase $\Gamma / \Omega$, the ESD regions shrink and the period of the entanglement oscillations becomes smaller. We note in passing that entanglement oscillations also occur when the qubits share just one excitation (as shown in Ref. [4]). In that case the memory depth of the reservoir leads to entanglement oscillations, but ESD does not occur.

Entanglement sudden death and entanglement revivals, in the common structured reservoir, are basically due to two combined and intertwined effects: the backaction of the structured reservoir and the reservoir-mediated interaction between the qubits. In order to understand the role played by each of these effects we compare our results with the cases of the common Markovian reservoir, and two independent non-Markovian reservoirs.

In Fig. 1(b) we show the entanglement dynamics for two qubits in a common Markovian reservoir. As explained in Ref. [10], a period of complete disentanglement is followed by a revival of entanglement, but no oscillations are present. Such a revival is due to the action of the common reservoir which tends to create quantum correlations between the qubits, providing an effective coupling between them. A comparison between Figs. 1(a) and 1(b) shows that the feedback of information from the reservoir into the system, characterizing non-Markovian dynamics, enhances the appearance of ESD regions, since it tends to recreate the conditions that led to the first ESD period.

Figure 1(c) shows the entanglement dynamics when the qubits interact with two independent non-Markovian reservoirs. Three different dynamical regions are clearly identified. Depending on the initial state of the system, entanglement can die after a finite time, or oscillate while going asymptotically to zero, or reappear after an ESD period. The
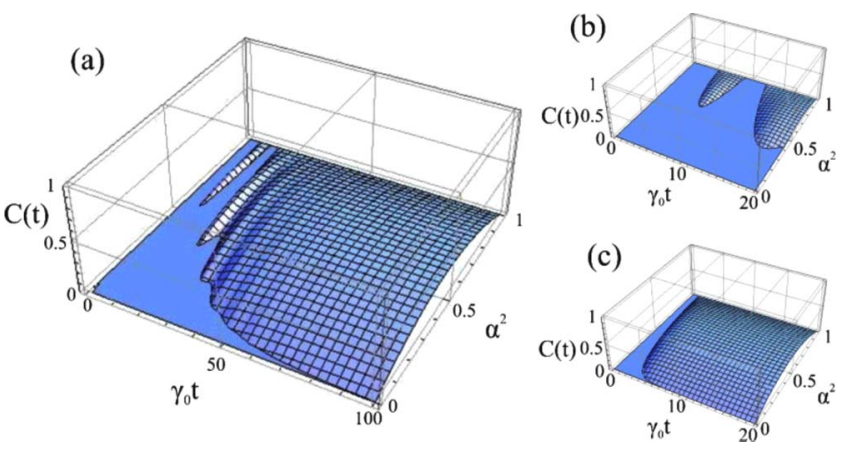

FIG. 2. (Color online) (a) Concurrence as a function of time and $\alpha^{2}$ for two atoms prepared in factorized state (10) and interacting with a common Lorentzian structured reservoir. For comparison we show the short-time region of (a) in (b), and the Markovian common reservoir result in (c).

revival phenomena in this case stem from the nonMarkovian behavior of each single qubit interacting with its own reservoir [3]. No revivals of entanglement are present in the Markovian case [8].

A comparison between the non-Markovian cases of Figs. 1(a) and 1(c) reveals that for the same type of reservoir spectrum, ESD regions are much wider in the independent reservoirs case than in the common reservoir case. Since both cases take into account memory effects, this suggests that the reservoir-mediated interaction between the qubits, in the common reservoir scenario, effectively counters the fast disappearance of entanglement.

Non-Markovian effects also influence strongly the dynamics for initially factorized states, when the reservoirmediated interaction between the qubits leads to entanglement generation. As an example we take the initial state

$$
\begin{aligned}
\rho(0)= & \left(\alpha^{2}\left|0_{A}\right\rangle\left\langle 0_{A}\left|+\left(1-\alpha^{2}\right)\right| 1_{A}\right\rangle\left\langle 1_{A}\right|\right) \otimes\left(\alpha^{2}\left|0_{B}\right\rangle\left\langle 0_{B}\right|\right. \\
& \left.+\left(1-\alpha^{2}\right)\left|1_{B}\right\rangle\left\langle 1_{B}\right|\right) .
\end{aligned}
$$

In the basis $\{|0\rangle,|+\rangle,|-\rangle,|2\rangle\}$ this state takes the form

$$
\begin{aligned}
\rho(0)= & \alpha^{4}|0\rangle\left\langle 0\left|+\left(1-\alpha^{2}\right)^{2}\right| 2\right\rangle\langle 2| \\
& +\alpha^{2}\left(1-\alpha^{2}\right)(|+\rangle\langle+|+|-\rangle\langle-|) .
\end{aligned}
$$

Again the density matrix has the $\mathrm{X}$ form so the concurrence can be calculated from Eq. (9). We evaluate the concurrence for the same parameters of the spectral distribution used in Fig. 1(a).

Figure 2 shows the evolution of concurrence when the atoms are prepared in factorized state (10). We note first that for independent reservoirs, sudden birth of entanglement does not appear, while in our common reservoir case, ESB does occur, as clearly demonstrated in Fig. 2(a). Moreover, in a structured reservoir such a phenomenon presents interesting features, compared to the Markovian case, as shown in Figs. 2(b) and 2(c). To elaborate: in a Markovian reservoir, entanglement sudden birth takes place at different times depending on the parameter $\alpha^{2}$. The smaller the value of $\alpha^{2}$ is, the longer is the time taken for entanglement generation. In other words, the ESB time monotonically decreases with $\alpha^{2}$. However, in the non-Markovian case, ESB revivals occur 
and complicate the simple Markovian picture. In general, the reservoir memory prolongs the initial disentanglement. In this case, therefore, the reservoir backaction dominates over the tendency of the common reservoir to create entanglement between the qubits. ESB periods and disentanglement revivals become more frequent and numerous for stronger nonMarkovian conditions.

It is worth noting that a necessary condition for ESB is that $\langle-|\rho|-\rangle \neq 0$. Indeed, the long-time asymptotic value of concurrence is directly related to the subradiant state component of the initial state [12].

Whatever the value of $\alpha$ is, for $t \rightarrow \infty$ the initial population of the three-state ladder system will decay to the $|0\rangle$ state, while the population of the subradiant $|-\rangle$ state will be trapped. The asymptotic stationary state of the system has the form

$$
\rho(t \rightarrow \infty)=(1-k)|0\rangle\langle 0|+k|-\rangle\langle-|,
$$

with $k=\alpha^{2}\left(1-\alpha^{2}\right)$. For $\alpha^{2} \neq 0$, or 1 , this state is not a factorized state. The stationary value of the concurrence, calculated from the analytic solution, is $C(t \rightarrow \infty)=k$.

In conclusion, we have presented a non-Markovian model describing the exact entanglement dynamics of two qubits interacting with a common structured reservoir. We have brought to light different entanglement features for qubits prepared in both entangled and factorized states. The nonMarkovian nature of the reservoir protracts the disentanglement process while enriching the revivals, and at the same time it enhances the regions of ESD. The backaction of the reservoir slows down the generation of entanglement and further manifests itself in the appearance of periods of death and resurrection. The reservoir-mediated interaction between the qubits strikingly distinguishes the dynamics in a common reservoir from the independent reservoirs case. Our predictions apply to cavity QED experiments with trapped ions, and to circuit QED experiments. In the first context, entanglement between two remotely located trapped atomic ions has been recently demonstrated [18] and multiparticleentangled states can be generated and fully characterized via state tomography [19]. In the second context, field coupling and coherent quantum state storage between two Josephson phase qubits have been achieved through a microwave cavity on chip $[17,20]$. Due to the possibilities for realizing strongcoupling conditions between atoms and a high finesse cavity [21], deep understanding of the non-Markovian dynamics is now indispensable.

The authors thank CIMO, the Academy of Finland (Projects No. 108699, No. 115682, and No. 115982), and the Turku University Foundation for support.
[1] S. Haroche and J.-M. Raimond, Exploring the Quantum: Atoms, Cavities, and Photons (Oxford University Press, Oxford, 2006).

[2] M. A. Nielsen and I. L. Chuang, Quantum Computation and Quantum Information (Cambridge University Press, Cambridge, England, 2000); S. Stenholm and K.-A. Suominen, Quantum Approach to Informatics (Wiley, Hoboken, NJ, 2005).

[3] B. Bellomo, R. Lo Franco, and G. Compagno, Phys. Rev. Lett. 99, 160502 (2007).

[4] S. Maniscalco, F. Francica, R. L. Zaffino, N. Lo Gullo, and F. Plastina, Phys. Rev. Lett. 100, 090503 (2008).

[5] B. Bellomo, R. Lo Franco, S. Maniscalco, and G. Compagno, Phys. Rev. A 78, 060302(R) (2008).

[6] H.-P. Breuer and F. Petruccione, The Theory of Open Quantum Systems (Oxford University Press, Oxford, 2002).

[7] J. León and C. Sabín, Phys. Rev. A 79, 012301 (2009).

[8] T. Yu and J. H. Eberly, Phys. Rev. Lett. 93, 140404 (2004).

[9] M. P. Almeida et al., Science 316, 579 (2007).
[10] Z. Ficek and R. Tanaś, Phys. Rev. A 74, 024304 (2006).

[11] D. Braun, Phys. Rev. Lett. 89, 277901 (2002); M. S. Kim, J. Lee, D. Ahn, and P. L. Knight, Phys. Rev. A 65, 040101(R) (2002); J. P. Paz and A. J. Roncaglia, Phys. Rev. Lett. 100 , 220401 (2008)

[12] Z. Ficek and R. Tanaś, Phys. Rev. A 77, 054301 (2008).

[13] C. E. Lopez, G. Romero, F. Lastra, E. Solano, and J. C. Retamal, Phys. Rev. Lett. 101, 080503 (2008).

[14] B. M. Garraway, Phys. Rev. A 55, 2290 (1997).

[15] B. J. Dalton and B. M. Garraway, Phys. Rev. A 68, 033809 (2003); B. J. Dalton, S. M. Barnett, and B. M. Garraway, ibid. 64, 053813 (2001).

[16] W. K. Wootters, Phys. Rev. Lett. 80, 2245 (1998).

[17] M. A. Sillanpää et al., Nature (London) 449, 438 (2007).

[18] D. L. Moehring et al., Nature (London) 449, 68 (2007).

[19] H. Häffner et al., Nature (London) 438, 643 (2005).

[20] J. Majer et al., Nature (London) 449, 443 (2007).

[21] G. R. Guthöhrlein et al., Nature (London) 414, 49 (2001); A. Wallraff et al., ibid. 431, 162 (2004). 